\title{
REMARKS ON THE RESTORATION OF \\ OCCULTATION OBSERVATIONS
}

\author{
TERENCE J. DEEMING
}

Dept. of Astronomy, University of Texas, Austin, Texas 78712, U.S.A.

If the limb of the Moon can be regarded as a straight edge, then the diffraction pattern of a point source which it produces at the distance of the Earth is the well known Fresnel diffraction pattern. Observations of stellar occultations reveal the variation of intensity with time as the diffraction pattern passes across the detector due to the orbital motion of the Moon and the rotation of the Earth. The linear scale of the diffraction pattern in monochromatic light depends on both the wavelength of observation, $\lambda$, and on the distance of the Moon, $D$, so that the scale is proportional to $(\lambda D)^{1 / 2}$. At a given wavelength, the diffraction pattern has the shape

$$
f_{p}(x, \lambda)=p\left(\frac{x}{(\lambda D)^{1 / 2}}\right)
$$

where $p(\xi)$ is a universal function (the Fresnel function) of its dimensionless argument. The subscript $p$ denotes the observation of a point source. If the velocity of the detector across the pattern is $v$, the distribution of intensity as a function of time will be

$$
f_{p}(t, \lambda)=p\left(v t /(\lambda D)^{1 / 2}\right) .
$$

$\xi=v t /(\lambda D)^{1 / 2}$ is therefore the natural, or dimensionless, unit of the problem.

For an extended source with brightness distribution $I(\theta)$, the observed occultation curve in monochromatic light will be the convolution of the point source occultation curve, $f_{p}(t, \lambda)$, with $I(\theta)$, although in writing this we have to change the variable $\theta$ to a time variable $\tau$ by the scaling

$$
\tau=\theta / \omega=\theta D / v
$$

where $\omega=v / D$ is the angular velocity of the Moon's limb as seen by the observer. The observed occultation curve of an extended source is then

$$
f(t, \lambda)=f_{p}(t, \lambda) * I(\tau)=\int_{-\infty}^{+\infty} p\left(\frac{v(t-\tau)}{(\lambda D)^{1 / 2}}\right) I(\tau) \mathrm{d} \tau .
$$

The aim of restoration, or deconvolution, techniques is of course to find a function $r(t, \lambda)$ which can be convolved with the observed pattern $f(t, \lambda)$ to yield an estimate of the original brightness distribution $I(\tau)$. It was shown by Scheuer (1962) that in theory the function

$$
r(t, \lambda)=p^{\prime \prime}\left(-v t /(\lambda D)^{1 / 2}\right)
$$


is a restoring function, $p^{\prime \prime}$ being the second derivative of the Fresnel function $p(\xi)$. However the convolution integral is very badly behaved, and it is necessary to smooth the restoring function with some function such as a Gaussian to make the integral converge. The conclusion is that it is possible to achieve, not a complete restoration, but a restoration to a brightness distribution which would have been observed by a system having a finite resolving power given essentially by the width of the smoothing function.

The value of the detector velocity, $v$, which is needed for this restoration process can be obtained from the lunar ephemeris and the position angle of the occultation. However, there may be a deviation of the local slope of the limb at the point of occultation giving some uncertainty as to the precise effective value of $v$ in the direction perpendicular to the actual occulting limb. But the function $p(\xi)$ is of course known theoretically, so that from strictly monochromatic observations of a point source, $f_{p}(t, \lambda)$, it is possible to determine an experimental value of the scale factor $v /(\lambda D)^{1 / 2}$. Since $\lambda$ and $D$ are known, as well as the expected limb velocity from the ephemeris and position angle of occultation, it is possible to compare observed and predicted velocity and so determine a slope for the lunar limb (Nather and Evans, 1970). In finding a restored brightness distribution, it is necessary to know the value of $v$, both to substitute into Equation (5) for the restoring function, and to convert from $\theta$ to $\tau$ according to Equation (3). Therefore the value of this local slope must be known. For sources which are not too different from point sources it should still be possible to fit a theoretical point source diffraction pattern to the observations, and so determine the scale factor and limb slope. However there is some error involved in this, and for objects of large angular size the fit to a point source curve to determine the scale factor may be grossly inaccurate. The alternatives are either to accept the ephemeris value of limb velocity and to ignore the slope of the lunar limb, or to make models of the theoretical occultation curves for assumed brightness distributions and try to match the observed occultation curves to them. This latter method, which is probably the best if plausible assumptions about the brightness distribution can be made, has been used successfully by Nather and Evans (1970). However, if we wish to pursue the idea of obtaining brightness distributions by direct restoration, it should be clear that some error is introduced by the unknown lunar limb slope, and that it is to some extent possible to trade lunar limb slope for angular diameter. A recent discussion of lunar limb irregularities has been given by Evans (1970).

The situation in practical optical observations is somewhat more complicated than described above, and several effects serving to degrade the visibility of the fringes need to be taken into account:

(i) Finite detector size. At a wavelength of $5000 \AA$ and a typical lunar distance of $3.8 \times 10^{10} \mathrm{~cm}$, the value of $(\lambda D)^{1 / 2}$ is about $14 \mathrm{~m}$. Since the higher order fringes of interest may have separations, in natural units, of about $\Delta \xi=0.1$ to 0.2 , or 1 to $3 \mathrm{~m}$, it follows that a $100 \mathrm{in}$. $(2.8 \mathrm{~m})$ aperture will produce a significant smoothing of the fringe pattern, at least in the higher order fringes.

(ii) Finite integration time. Practical considerations require the observation of 
$f(t, \lambda)$ using a small but finite integration time, $t$, so that what is actually observed is an average quantity

$$
f(t, \lambda)=\frac{1}{\Delta t} \int_{t}^{t+\Delta t} f(t, \lambda) \mathrm{d} t
$$

With a typical limb velocity of $v \simeq 1 \mathrm{~km} \mathrm{sec}^{-1}=1 \mathrm{~m} \mathrm{msec}^{-1}$, the factor $(\lambda D)^{1 / 2} / v$ is about $14 \mathrm{msec}$. Observations by Nather and Evans (1970) involve integration times of about $1-2 \mathrm{msec}$, so this does not appear to contribute significantly to the degradation of fringe visibility although clearly it sets a limit to the brightness of stars which can be observed.

(iii) Finite observing time. Since observations do not continue for an infinite time but exist only over a time interval $(O, T)$, restoration cannot take place through an infinite integral. This has the effect of smoothing the theoretical brightness distribution with the convolution of the 'data window' $(O, T)$ and the restoring function.

(iv) Finite bandwidth. Optical observations are usually not monochromatic, nor even quasi-monochromatic, but cover quite a wide range of wavelengths. The $B$ filter of the UBV system, for instance, has a bandwidth $\delta \lambda / \lambda$ of about 0.1 . Under these circumstances, the diffraction pattern of a point source is a smoothed Fresnel function:

$$
f_{p}(t)=\int_{0}^{\infty} S(\lambda) E(\lambda) f_{p}(t, \lambda) \mathrm{d} \lambda=\int_{0}^{\infty} S(\lambda) E(\lambda) p\left(\frac{v t}{(\lambda D)^{1 / 2}}\right) \mathrm{d} \lambda
$$

where $S(\lambda)$ is the sensitivity function of the detector system, and $E(\lambda)$ is the spectral energy distribution of the star. This smoothing is not a convolution in the usual sense, and is not equivalent to the convolution of the monochromatic diffraction pattern with any physically possible aperture function. However, if we allow physically impossible aperture functions, involving negative responses, then it is possible to convert Equation (7) into the form of a convolution by the following mathematical device: Take the Fourier transform of Equation (7) so that the transform of $f_{p}(t)$ is $F_{p}(v)$. Now pick some wavelength, $\lambda_{0}$, and let the Fourier transform of the monochromatic point source diffraction pattern, $f_{p}\left(t, \lambda_{0}\right)$ be $F_{p}\left(v, \lambda_{0}\right)$. Then

$$
F_{p}(v)=F_{p}\left(v, \lambda_{0}\right) \frac{F_{p}(v)}{F_{p}\left(v, \lambda_{0}\right)}=F_{p}\left(v, \lambda_{0}\right) R\left(v, \lambda_{0}\right)
$$

defining $R\left(v, \lambda_{0}\right)$ which is a function only of the frequency $v$ and the chosen wavelength $\lambda_{0}$. Now Fourier transform back to the $t$ domain, remembering that the Fourier transform of a product is a convolution, and obtain

$$
f_{p}(t)=f_{p}\left(t, \lambda_{0}\right) * r\left(t, \lambda_{0}\right)
$$

where $r\left(t, \lambda_{0}\right)$ is the Fourier transform of $R\left(v, \lambda_{0}\right)$. This equation merely says that, provided the Fourier transforms exist, any function $f(t)$ can be expressed as the convolution of any other function $f_{p}\left(t, \lambda_{0}\right)$ with some function $r\left(t, \lambda_{0}\right)$. This in itself 
says nothing about the nature of the function $r\left(t, \lambda_{0}\right)$ although clearly it can be evaluated given $S(\lambda)$ and $E(\lambda)$. Khrishnan (1970) has evaluated $r\left(t, \lambda_{0}\right)$ for various forms of $S(\lambda)$ and has concluded that it resembles in general character an aperture smoothing function, or Gaussian function, and suggests that the finite bandwidth thereby provides the necessary smoothing of the restoring function $p^{\prime \prime}(-\xi)$, and that it is not necessary to use any additional smoothing.

(v) Noise. As with any set of experimental data, the noise (observational uncertainty) due to various causes provides some limitation on the resolution achievable. Clearly a given level of noise will effectively wipe out fringes beyond a certain point, since higher order fringes have successive smaller amplitudes. This may be expected to have an effect similar to that of a finite time interval of observation. There is not much point in observing beyond the point where the fringes have been lost in the noise.

All of these effects, with the possible exception of the noise, represent a degradation of the diffraction pattern in the sense that, while the theoretical, infinite, monochromatic diffraction pattern contains information about all the Fourier components of the source brightness distribution in the direction of the occultation, the observed diffraction pattern has these Fourier components either modified or eliminated.

There is a way of looking at the problem which at first sight appears very simple. If we are given the diffraction pattern $f_{p}(t)$ which is produced by a point source with any given system of observation, (including given $v, D, S(\lambda), E(\lambda)$, telescope aperture, etc.), then it is generally true that the diffraction pattern of an extended source with strip brightness distribution (in $\tau$ units) $I(\tau)$ will be the convolution of $f_{p}(t)$ with $I(\tau)$ :

$$
f(t)=\int_{-\infty}^{+\infty} f_{p}(t-\tau) I(\tau) \mathrm{d} \tau .
$$

This convolution equation is generally true, regardless of the form of $f_{p}(t)$. Such a convolution equation may always be solved, in principle, either by Fourier transform techniques, or by some other method. We may, then, reach the conclusion that apart from the problem of noise on the data, a solution is possible giving an exact restoration, not subject to loss of resolution due to bandwidth or similar effects.

A difficulty is that the solution to a convolution equation is not necessarily unique. In particular, if the Fourier transform of the function $f_{p}(t)$ has any zeros at some frequencies $v_{1}, v_{2}, \ldots$, the Fourier components of $I(\tau)$ at these frequencies will not contribute at all to the observed diffraction pattern, so certain parts of the brightness distribution are 'invisible' to the occultation. A similar effect is discussed by Bracewell and Roberts (1954) in connection with aerial smoothing in radio astronomy. The effect is also similar to that of aliasing in power spectrum analysis. While the exact nature of the non-uniqueness has not been fully investigated as yet for this particular problem, it has seemed worthwhile to pursue the business of obtaining at least $a$ solution, in the hope that physically plausible solutions can be separated from physically implausible ones. 
In practical observations an integration time $\Delta t$ is required to obtain significant numbers of photons, and observations are only carried out within some finite time interval $(O, T)$. Values of $f(t)$ are therefore not available continuously, but only at a finite set of $N$ discrete times, $t_{i}$. We may therefore write Equation (10) in the discrete form

$$
f_{i}=\sum_{k=1}^{N} f_{P_{i k}} I_{k}
$$

where we have used the following notations and substitutions:

$$
\begin{aligned}
& f_{i}=f\left(t_{i}\right) \quad f_{P_{i k}}=f_{p}\left(t_{i}-\tau_{k}\right) \\
& I_{k}=I\left(\tau_{k}\right) \quad N=T / \Delta t .
\end{aligned}
$$

We have assumed here that we are able to recover $N$ values of $I(\tau)$. In view of the fact that we have only $N$ data points, it is certainly inadvisable to try to recover more than $N$ values. We might ask for fewer than $N$ values and treat the problem in a least squares way, but in order to do this some further assumptions about the form of the brightness distribution $I(\tau)$ would have to be made. For the present, and for the sake of simplicity, we just try to recover the maximum number, $N$, of values of $I(\tau)$. In choosing to determine $I(\tau)$ only at certain discrete values of $\tau$, we are in effect ignoring certain Fourier components of $I(\tau)$. Undoubtedly some, and perhaps all, of these will be frequencies about which no information could be obtained in any case because of the non-uniqueness of the deconvolution discussed above.

Equation (11) is a matrix equation which, in principle, can be inverted to give a matrix $r_{j i}=\left(f_{p}^{-1}\right)_{j i}$ so that $r_{j i}$ becomes a restoration matrix which will restore completely the original brightness distribution at $N$ points $\tau_{j}$ :

$$
\sum_{i} \tau_{j i} f_{i}=I_{j}
$$

Since the restoration is effected by a simple linear operation (matrix multiplication) it should also be fairly straightforward to compute the expected effects of noise in the data - an important point in assessing the significance of features in a restored curve.

With regard to the reduction of Equation (10) to Equation (11), it should also be remarked that there arise 'edge effects' from treating the convolution as a finite sum rather than an infinite integral. Provided, however, that $I(\tau)$ is reasonably concentrated near $\tau=0$ this should not matter. If $I(\tau)$ is not so concentrated, then the information needed for solution is not contained in the finite data and no technique will reveal it. Again this restriction is equivalent to ignoring certain Fourier components of $I(\tau)$.

The computation time (and storage space) required for the inversion of large matrices is quite large, increasing roughly as $N^{3}$. For experiments, a $50 \times 50$ matrix is fairly easy to handle with the CDC 6600 computer at the University of Texas, and seems to be representative of the kind of data available at present. In several tests 
we have found that the matrix $f_{p}$ is indeed not singular, and that inversion takes about $15 \mathrm{sec}$. The success of this implies that in these particular cases, the Fourier transform of $f_{p}(t)$ has no zeros at the frequencies corresponding to multiples of the data spacing, $\Delta t$. It is possible that in certain unlucky circumstances we may pick a data spacing in which this is not true.

Experiments on the use of the matrix inversion method of restoration are continuing. It proves to be fairly simple to analyse the effects of observational noise on the restored brightness distribution; the effect appears to be strongly asymmetrical across the restored distribution and to show a fairly high autocorrelation.

A practical problem arises in the actual definition of the diameter of the restored brightness distribution; once we choose to fit a model distribution - say a uniform or limb-darkened disc - then we would be better off fitting a model to the original occultation curve where the effect of observational error is clearer. Probably the ideal compromise is the use of deconvolution (restoration) as an investigative tool, followed by model fitting to determine numerical parameters.

I would like to thank D. S. Evans and R. E. Nather for many illuminating discussions.

\section{References}

Bracewell, R. N. and Roberts, J. A.: 1954, Australian J. Phys. 7, 615.

Evans, D. S.: 1970, Joint Discussion on Occultations, XIV IAU, Brighton.

Khrishnan, T.: 1970, NASA Technical note D-5679.

Nather, R. E. and Evans, D. S.: 1970, Astron. J. 75, 575.

Scheuer, P. A. G.: 1962, Australian J. Phys. 15, 333. 